\title{
ORIGINAL RESEARCH \\ Coiling for Paraclinoid Aneurysms: Time to Make Way for Flow Diverters?
}

\author{
P.I. D’Urso \\ H.H. Karadeli \\ D.F. Kallmes
}

H.J. Cloft

G. Lanzino

\begin{abstract}
BACKGROUND AND PURPOSE: Paraclinoid aneurysms represent challenging lesions for which endovascular techniques have gained widespread application in their treatment. A new endovascular strategy, flow diversion, is gaining importance in the treatment of these aneurysms. Before embracing flow diversion in larger numbers of patients with paraclinoid aneurysms, it is important to know the safety and efficacy of "traditional" endovascular methods for these aneurysms. We review complications and outcomes of patients with unruptured paraclinoid aneurysms treated with coils, with or without balloon and stent assistance, over the past 12 years at our institution.
\end{abstract}

MATERIALS AND METHODS: A retrospective review of 118 patients with 126 unruptured paraclinoid aneurysms, treated between 1999 and 2010, was performed. Clinical records, endovascular reports, angiographic results, and clinical outcomes were reviewed and analyzed.

RESULTS: Fifty-nine percent of aneurysms were carotid-ophthalmic, 27\% were hypophyseal, 9\% were posterior carotid wall, $3 \%$ were carotid cave, and $2 \%$ were transitional. Twenty-one percent of aneurysms were symptomatic, and $3 \%$ were recurrent aneurysms after previous treatment. Fifty $(40 \%)$ were treated with balloon assistance and $18(14 \%)$ with stent assistance. Technical complications causing permanent morbidity occurred in 1 patient $(0.8 \%)$, while early clinical complications causing transient morbidity occurred in $5(4 \%)$ patients. Complete occlusion was achieved in $40 \%$ of aneurysms immediately after treatment and in $66(62 \%)$ aneurysms during follow-up (mean $31.9 \pm$ 28.4 months). Recurrences occurred in 18 patients $(17 \%)$ and $10(9 \%)$ patients were retreated. Clinical outcome was good in $95 \%$ and poor in $5 \%$ of the 107 patients with follow-up (mean $37.0 \pm 33.7$ months). Only in 1 patient was poor outcome related to the endovascular procedure. Transient ischemic attacks occurred in $4 \%$ of patients. No cases of rebleeding were observed during follow-up.

CONCLUSIONS: Modern endovascular coil treatment of unruptured paraclinoid aneurysms appears safe, with satisfactory, although not ideal, rates of complete occlusion. The introduction of flow diverters will probably increase the rate of complete angiographic occlusion, but this must be achieved with low complication rates.

ABBREVIATIONS: $\mathrm{Cl}=$ confidence interval; $\mathrm{OR}=$ odds ratio; $\mathrm{mRS}=$ modified Rankin Scale; $\mathrm{VP}=$ ventriculoperitoneal
$\mathbf{P}$ araclinoid aneurysms are lesions located adjacent to the clinoid segment of the ICA. ${ }^{1}$ Microsurgical treatment of these aneurysms is challenging because of their proximity to optic apparatus, bone structures, and cavernous sinus. Accordingly, endovascular techniques have gained widespread application in their treatment. Despite availability of adjunctive techniques, such as balloon-assisted and stent-assisted coiling, coil embolization continues to be associated with a high rate of residual/recurrent aneurysm filling. In the past few years, flow diverters have been introduced as an alternative and more effective endovascular technique than coil embolization, and in April 2011, the Pipeline Embolization Device (Chestnut Medical Technologies, Menlo Park, California) was approved by the FDA for treatment of large or giant wide-neck intracranial aneurysms in the proximal intracranial ICA, including the ophthalmic segment. It is conceivable that as more experience is gained, flow diverters may be utilized for more "routine" aneurysms involving the proximal segment of the ICA. Before flow diverters are adopted on a large scale, it is

Received June 6, 2011; accepted after revision November 30.

From the Departments of Neurosurgery (P.I.D., H.H.K., G.L.) and Radiology (D.F.K., H.J.C., G.L.), Mayo Clinic, Rochester, Minnesota.

Please address correspondence to Giuseppe Lanzino, Department of Neurosurgery, Mayo Clinic, 200 First Street, Rochester, MN 55905; e-mail: Lanzino.Giuseppe@mayo.edu

http://dx.doi.org/10.3174/ajnr.A3009 important to know the efficacy and safety of current endovascular techniques for the treatment of these aneurysms. For this purpose, we retrospectively reviewed our single-center experience with coiling of consecutive patients with unruptured paraclinoid aneurysms.

\section{Materials and Methods}

After institutional review board approval, a retrospective review of patients treated with coil embolization at our institution (Mayo Clinic, Rochester, Minnesota) was performed. Between January 1999 and October 2010, 772 patients underwent endovascular treatment for 855 intracranial aneurysms, including 136 patients with 149 unruptured aneurysms (31\%) in the paraclinoid region. Paraclinoid aneurysms were defined as those aneurysms located in the intradural paraclinoid area, proximal to the origin of the posterior communicating artery. Aneurysms were classified into transitional, carotid cave, carotid-ophthalmic, superior hypophyseal, and posterior carotid wall aneurysms. ${ }^{1}$ Fourteen aneurysms treated with flow diverters were excluded from the present series. Similarly, patients with large and giant aneurysms who underwent parent artery sacrifice with or without surgical bypass revascularization and patients with dissecting aneurysms were excluded from analysis. Clinical and radiographic data on the remaining 118 patients with 126 unruptured paraclinoid aneurysms were extracted from our institutional electronic medical data base. Aneu- 


\begin{tabular}{|c|c|c|c|}
\hline & $\begin{array}{l}\text { Unruptured } \\
\text { Paraclinoid }\end{array}$ & $\begin{array}{l}\text { Unruptured Other } \\
\text { Locations }\end{array}$ & $P$ \\
\hline \multicolumn{4}{|l|}{$\overline{\text { Sex }}$} \\
\hline Female & $109(92 \%)$ & $70 \%$ & \multirow[t]{3}{*}{$<.001^{*}$} \\
\hline Male & $9(8 \%)$ & $30 \%$ & \\
\hline Female:Male & $12.6: 1$ & $2.4: 1$ & \\
\hline Mean age (yr) & $55.19 \pm 11.7(30-79)$ & $57.5 \pm 12.8$ & $.030^{*}$ \\
\hline \multicolumn{4}{|l|}{ Risk factors } \\
\hline Hypertension & $53(45 \%)$ & $55 \%$ & .076 \\
\hline Diabetes mellitus & $5(4 \%)$ & $9 \%$ & .080 \\
\hline \multicolumn{4}{|l|}{ Tobacco abuse } \\
\hline Current & $35(30 \%)$ & $39 \%$ & \multirow[t]{2}{*}{.115} \\
\hline Prior & $34(29 \%)$ & $30 \%$ & \\
\hline Personal history of cerebrovascular disease & $16(14 \%)$ & $19 \%$ & .222 \\
\hline Family history of intracranial aneurysm & $34(29 \%)$ & $15 \%$ & $.001^{*}$ \\
\hline Multiple aneurysms & $47(40 \%)$ & $33 \%$ & .271 \\
\hline Length of hospital stay (days; mean \pm SD) & $1.54 \pm 2.6$ & $1.9 \pm 2.9$ & .080 \\
\hline
\end{tabular}

${ }^{*}$ Statistically significant.

rysm size was categorized as small $(<10 \mathrm{~mm})$, large $(10-24 \mathrm{~mm})$, and giant $(\geq 25 \mathrm{~mm})$.

Information collected included patient demographics, risk factors for intracranial aneurysms (including prior or current history of smoking, history of hypertension, family history of intracranial aneurysms), and length of hospital stay. The total number of aneurysms identified on cerebral angiogram, along with location and size of each, as well as information about treatment and eventual use of balloon or stent assistance were also abstracted. Preoperative and peri- and postprocedural treatment with antiplatelet drugs was recorded.

Aneurysm occlusion was graded using the 3-point modified Raymond scale. ${ }^{2}$ Information about radiologic (DSA and/or MRA) follow-up was recorded for every aneurysm at various intervals and at the last radiologic follow-up. Recurrence was defined as a change in degree of occlusion from a Raymond class to a higher one. Recurrences were further subdivided into those requiring retreatment and those managed conservatively. Clinical outcomes were reported using the $\mathrm{mRS}^{3,4}$ and dichotomized into "good" (mRS 0-2) and "poor" (mRS 3-6) outcomes.

Technical complications, including perforation, coil prolapse, coil migration, endoluminal thrombus formation, and distal emboli, were recorded. Coil prolapse was subsequently defined as no-flow limiting and flow limiting, and information was acquired concerning whether or not any intra-arterial thrombolysis was used. Complications were considered "periprocedural" if manifest during the procedure, "early" if occurring within 30 days from the procedure, and "delayed" if occurring after the 30-day time interval. Early clinical complications were defined as any thromboembolic, hemorrhagic, or nonneurologic complication occurring within 1 month after endovascular treatment. Complications (technical and clinical) accounting for permanent morbidity or mortality were used to define procedural morbidity and procedural mortality.

Clinical data collected at the last follow-up included information regarding any occurrence of rebleeding and possible delayed ischemic symptoms related to possible thromboembolism induced by the coils and/or stent. Variation of the mRS scores at follow-up compared with preprocedural scores - and, when applicable, causes of worseningwere analyzed.

Subgroup analyses were performed based on age, aneurysm location (paraclinoid classification), size, and use of balloon and/or stent placement during the treatment. Furthermore, a subgroup analysis comparing aneurysms larger than $10 \mathrm{~mm}$ to those smaller than 10 $\mathrm{mm}$ was performed to analyze potential differences in the rate of technical/clinical complications, occlusion grade, and recurrence/retreatment between the 2 groups. When appropriate, comparisons were made with unruptured aneurysms in other locations treated at our institution during the same time interval.

The $\chi^{2}$ test, Student $t$ test, Fisher exact test, and Kruskall-Wallis test were used, as appropriate. Correlation between age, dome size, technical complications, early and delayed clinical complications, balloon and stent assistance, and final outcome were studied with univariate analyses, and variables with significant $P$ values $(P<$ $.05)$ were chosen as variables for multiple logistic regression analyses. Tests were conducted as 2 -sided, at a statistical significance level $(P)$ of .05. Continuous data are presented as mean \pm standard deviation. All statistical analyses were performed with JMP software, version 8.0.

\section{Results}

Patient demographics and general clinical information are summarized in Table 1. One hundred nine patients (92\%) were female and 9 patients were male (8\%); 47 patients (40\%) had multiple aneurysms, including 36 (31\%) with bilateral paraclinoid aneurysms. Mean age at presentation was $55.1 \pm$ 11.7 years. Compared with patients with unruptured intracranial aneurysms in other locations treated with endovascular therapy at our institution, patients with paraclinoid aneurysms were more frequently women $(P<.001)$ and of younger age $(P=.025)$.

Forty-five percent of patients were given prophylactic (in preparation for endovascular treatment) antiplatelet therapy (31\% single and 14\% double antiplatelet therapy).

Seventy-four aneurysms (59\%) were classified as carotidophthalmic, $34(27 \%)$ as superior hypophyseal, $11(9 \%)$ as posterior carotid wall, $4(3 \%)$ as carotid cave, and $3(2 \%)$ as transitional (Table 2). One hundred three aneurysms $(82 \%)$ were small, $22(17 \%)$ were large, and 1 was giant $(0.8 \%)$; the overall largest diameter mean was $7.3 \pm 3.6 \mathrm{~mm}$. All aneurysms were saccular, while 58 aneurysms (51\%) were wideneck (neck $>4 \mathrm{~mm}$ ). Four of the treated aneurysms (3\%) were 


\begin{tabular}{lcccc}
\hline Table 2: Aneurysms characteristics & & & \\
\hline & & & Size & \\
\cline { 2 - 5 } Aneurysm Location & Small $(<10 \mathrm{~mm})$ & Large $(10-25 \mathrm{~mm})$ & Giant $(>25 \mathrm{~mm})$ \\
\hline Carotid-ophthalmic & 58 & 15 & 1 & 0 \\
Superior hypophyseal & 32 & 2 & 0 & $74(59 \%)$ \\
Posterior carotid wall & 8 & 3 & 0 & $34(27 \%)$ \\
Carotid cave & 4 & 0 & 0 & $4(3 \%)$ \\
Transitional & 1 & 2 & $1(0.8 \%)$ & $3(2 \%)$ \\
Total & $103(82 \%)$ & $22(17 \%)$ & 126 \\
\hline
\end{tabular}

\begin{tabular}{|c|c|c|c|c|}
\hline & Observed & $\begin{array}{l}\text { Transient } \\
\text { Morbidity }\end{array}$ & $\begin{array}{c}\text { Permanent } \\
\text { Morbidity }\end{array}$ & Mortality \\
\hline \multicolumn{5}{|l|}{ Technical complications } \\
\hline Perforation & $4(3 \%)$ & $1(0.8 \%)$ & - & - \\
\hline Distal emboli & $4(3 \%)$ & - & $1(0.8 \%)$ & - \\
\hline Coil prolapsing flow limiting & $3(2 \%)$ & - & - & - \\
\hline Collateral branches occlusion & $1(0.8 \%)$ & - & - & - \\
\hline Thrombus formation & $6(5 \%)$ & - & - & - \\
\hline Catheter-induced vasospasm & $2(1.6 \%)$ & - & - & - \\
\hline \multicolumn{5}{|l|}{ Early clinical complications } \\
\hline Thromboembolic complications & $5(4 \%)$ & $5(4 \%)$ & - & - \\
\hline
\end{tabular}

recurrences after prior treatments ( 1 coiling, 1 stent-assisted coiling, 1 clipped, 1 clipped and stent-assisted coiled), and 8 patients $(6 \%)$ had a prior history of SAH from another aneurysm.

Most of the treated aneurysms were incidental (100 aneurysms, 79\%), while 26 aneurysms (21\%) were symptomatic. Headache $(10 \%)$ and visual symptoms (9\%) were the most common presenting symptoms. Visual symptoms prevailed in patients harboring carotid-ophthalmic aneurysms, and, in 3 patients with aneurysms $>10 \mathrm{~mm}$, were probably related to the mass effect of the aneurysmal sac. In 3 cases, aneurysms manifested with seizures $(2 \%)$, and in 1 case $(0.8 \%)$, transient ischemic attack.

\section{Treatment}

All aneurysms were treated with coil embolization. In 54\% of the aneurysms treated with coil embolization, balloon assistance $(40 \%)$ or stent assistance $(14 \%)$ was utilized. Balloon and stent assistance were more commonly utilized in paraclinoid aneurysms than in unruptured aneurysms in other locations (40\% and $14 \%$ versus $19 \%$ and $7 \%$, respectively; $P$ s $<$ .001 ), and these adjunct techniques were not associated with a higher incidence of early or delayed thromboembolic complications $(P=.671$ and $P=.565$, respectively).

\section{Technical Complications}

Technical complications included 4 cases of aneurysm perforation (3\%), 4 cases of distal embolism (3\%), 3 cases of coil prolapse with partial limitation of flow into the parent vessel $(2 \%)$, and 1 case of branch artery occlusion $(0.8 \%)$. In the case of branch occlusion, a tiny marker of the catheter embolized distally without clinical sequelae. In 6 patients $(5 \%)$, thrombus formation was observed in the region of the aneurysm neck; in 3 of these 6 cases, the thrombus resolved spontaneously, and in the remaining 3 , after systemic and/or intra-arterial administration of glycoprotein IIb/IIIa inhibitors (abciximab [ReoPro] or eptifi- batide [Integrilin]). Catheter-induced vasospasm occurred in 2 cases $(1.6 \%)$. These technical complications caused transient morbidity in 1 patient $(0.8 \%)$, permanent morbidity in another $(0.8 \%)$ (Table 3$)$, and did not significantly affect final outcome $(P=.576)$. Transient morbidity was related to intraprocedural rupture during treatment of a coexistent unruptured superior cerebellar artery aneurysm. This complication required CSF diversion and a VP shunt for communicating hydrocephalus; at the last follow-up, this patient was neurologically intact. Permanent morbidity was observed in 1 patient $(0.8 \%)$. This 33-year-old woman, affected by a recurrent carotid-ophthalmic aneurysm coiled 4 months before in an outside facility, experienced visual loss in the right eye secondary to embolic occlusion of the central retinal artery. No cases of periprocedural mortality were observed. Subgroup analysis comparing aneurysms $>10 \mathrm{~mm}$ with those $<10 \mathrm{~mm}$ did not reveal significant differences in the rates of technical complications between the 2 groups $(P=.410)$.

\section{Early Clinical Complications}

Early complications following endovascular procedure occurred in 5 patients (4\%) (Table 3). These patients suffered thromboembolic complications, resulting in transient morbidity. Two patients experienced TIAs, and 2 patients experienced amaurosis fugax within 24 hours of the procedure. One patient, with pre-existing normal pressure hydrocephalus and VP shunt, and an incidental carotid-ophthalmic aneurysm, experienced mild transient left hemiparesis secondary to postprocedural embolic infarction, with complete recovery. Finally, overall periprocedural transient morbidity was $4.8 \%$, and periprocedural permanent morbidity was $0.8 \%$. No cases of periprocedural or early mortality were observed in this cohort of patients. No significant differences in the rates of early complications were observed after subgroup analysis of aneurysms larger or smaller than $10 \mathrm{~mm}(P=.481)$. 


\section{Angiographic Outcome}

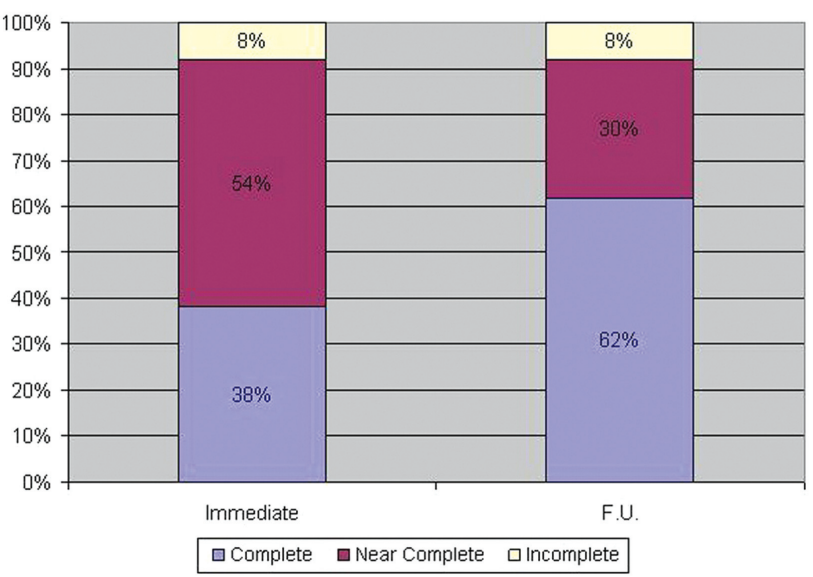

Fig 1. Angiographic results showing comparative results between immediate and delayed angiographic occlusion grade in the restricted subgroup of patients with radiologic follow-up. Occlusion grade scored with Raymond scale.

\section{Angiographic Results}

Immediate complete occlusion (Raymond class 1) was achieved in 50 aneurysms (40\%). Radiologic follow-up was available for 106 aneurysms (84\%), after an average time of $31.9 \pm 28.4$ months (range $2-125$ months). During the follow-up period, complete aneurysm occlusion was demonstrated in 66 aneurysms (62\%) (Fig 1). A nonsignificant trend toward higher degree of complete occlusion was observed in patients undergoing stent-assisted ( $81 \%$ versus $59 \%, P=.07$ ), balloon-assisted coiling ( $67 \%$ versus $59 \%, P=.44$ ). There was a statistically higher degree of complete occlusion in aneurysms $<10 \mathrm{~mm}$ compared with those $>10 \mathrm{~mm}$ (71\% versus $29 \%, P<.001)$.

Recurrences were observed in 18 aneurysms (17\%). Ten recurrences (55\% of the recurrent aneurysms and $9 \%$ of the 106 aneurysms with radiologic follow-up) were retreated with 1 or more procedures ( 9 recoilings and 1 stent-assisted recoiling). Recurrences were less common in patients treated with stent-assisted coiling compared with those in whom only coiling was performed ( $6 \%$ versus $19 \%, P=.171$ ), and were more frequently observed in older patients $(P=.013)$. Furthermore, the recurrence rate was significantly higher for aneurysms $>10 \mathrm{~mm}$ compared with aneurysms $<10 \mathrm{~mm}$ (33\% versus $13 \%, P=.025)$, though no significant differences were observed in the retreatment rate $(P=.083)$ between the 2 groups.

\section{Clinical Outcome}

Clinical follow-up was available for 107 patients (91\%) with 115 aneurysms. Average length of clinical follow-up was $37.0 \pm 33.7$ months (range $2-132$ months). In patients available for clinical follow-up, overall outcome was good in 102 patients (95\%) and poor in 5 patients (5\%). Reasons for poor outcome at follow-up included treatment-related visual impairment in 1 patient, worsening of the mRS for reasons not related to the treated aneurysm in 1 patient (arthritic knee), pre-existing (to endovascular treatment of the paraclinoid aneurysm) poor neurologic baseline in 2 patients, and 1 death occurring during the follow-up from complications related to the surgical treatment of a separate intracranial aneurysm in another patient. In 4 patients (4\%), TIAs occurred during the follow-up period, without permanent neurologic sequelae. No cases of rebleeding occurred during the follow-up period.

\section{Results of Statistical Analyses}

After univariate analysis, significant correlations were found between aneurysm size and age $(P<.001)$, aneurysm size and history of hypertension $(P<.001)$, aneurysm size and prior or current history of tobacco use $(P=.018)$, and aneurysm size and grade of initial $(P=.004)$ and final occlusion $(P=.005)$. Furthermore, grade of complete occlusion during the follow-up was correlated with the grade of initial aneurysm occlusion $(P=.001)$.

After logistic regression analysis, aneurysm size resulted in a significant predictive factor of initial (OR 1.14, $P=.008)$ and final (OR 1.18, $P=.001)$ incomplete occlusion. Furthermore, aneurysm size $>10 \mathrm{~mm}$ was found to be a strong predictor of incomplete occlusion (OR 5.99; 95\% CI, 2.17-18.50; $P<.001$ ) and recurrence (OR 3.36; 95\% CI, 1.07-10.1; $P=.036)$.

\section{Discussion}

In this large cohort of patients with midterm clinical and radiologic follow-up, coiling of unruptured paraclinoid aneurysms was technically feasible, with satisfactory rates of complete occlusion and low complication rates. In relation to the presence of the high percentage of wide-neck aneurysms, balloon or stent assistance was more frequently used compared with unruptured aneurysms in other locations in our institution dataset, and the use of these devices was not related to postprocedural thromboembolic events. Final occlusion rates in this series were in line with the reported range for these aneurysms of $50 \%$ to $88 \% .^{5-15}$ With current coil techniques (with or without adjuncts such as balloons and stents), treatment of most small paraclinoid aneurysms is very safe, as we have observed a very low incidence of permanent morbidity and no mortality in our series. Technical complications (including intraprocedural perforation in 5 patients) are not uncommon but did not affect outcome.

With the increasing use of flow diverters for the treatment of paraclinoid aneurysms, the question arises as to whether consistent use of these newer devices to treat "routine" paraclinoid aneurysms is as safe and effective as current standard endovascular techniques. Flow diverters seem to be theoretically indicated for the treatment of paraclinoid aneurysms because, in this region, there are not important perforators and covering the ophthalmic artery with a flow diverter seems to be well tolerated. ${ }^{16}$ Furthermore, in relation to its proximal location, the paraclinoid area is easily reached by flow diverters compared with more distal locations. However, the flow diversion technique is not devoid of complications, as reported from the recent published series in which complications were observed in up to $7.6 \%$ and mortality rates in up to $8 \%,{ }^{16}$ even if, in most of them, flow diverters were used for the treatment of complex intracranial aneurysms. The chance of complete occlusion with flow diverters, especially in side wall aneurysms like the paraclinoid type, is very high and most likely higher than coil embolization with or without balloon or stent assistance, though concerns related to the inability to immediately occlude the aneurysm exist, with the risk of aneurysm rupture during the "latency" period. In our series, recurrences were observed in $17 \%$ of the aneurysms treated, and in $9 \%$ of cases 
retreatment was considered indicated. However, it is questionable as to whether these recurrences are very clinically significant. Experience suggests that rupture of a previously coiled, unruptured, small paraclinoid aneurysm is exceedingly rare (not reported, to our knowledge), and no ruptures were observed in this and other series, ${ }^{15}$ except for very large and giant paraclinoid aneurysms. ${ }^{8}$

Our study has several limitations related primarily to the retrospective nature and the variable duration of follow-up. Moreover, most of the aneurysms included and treated were small aneurysms, which do not compare with the large and complex aneurysms that have been treated with first-generation flow diverters. Nevertheless, this series summarizes the results in a relatively homogeneous group of patients (with only unruptured aneurysms) and provides a modern (first patients were treated in 1999) snapshot indicating that standard endovascular techniques are both safe and clinically effective in treating less complex paraclinoid aneurysms.

\section{Conclusions}

Despite the limits related to the retrospective nature of the present study, our results confirm that coil embolization, with or without balloon or stent assistance, is a very safe and effective treatment for most routine unruptured paraclinoid aneurysms. Although flow diverters may increase the rate of complete angiographic occlusion, carefully conducted studies are required before "routine" application of these devices to small unruptured paraclinoid aneurysms is considered.

Disclosures: David F. Kallmes—RELATED: Grant: eV3, ${ }^{*}$ NFocus, ${ }^{*}$ MicroVention, ${ }^{*}$ Sequent, ${ }^{*}$ Penumbra, ${ }^{*}$ Micrus, ${ }^{*}$ Comments: Research support; UNRELATED: Payment for Development of Educational Presentations: eV3, CareFusion; Travel/Accommodations/Meeting Expenses Unrelated to Activities Listed: MicroVention. ${ }^{*}$ Harry J. Cloft-UNRELATED: Grants/Grants Pending: MindFrame. ${ }^{*}$ Giuseppe Lanzino—RELATED: Grant: eV3, ${ }^{*}$ Comments: Educational Grant for Journal Club and Visiting Professor Program; Support for Travel to Meetings for the Study or Other Purposes: eV3, ${ }^{*}$ Comments: To present to FDA panel; UNRELATED: Educational grant: Synthesis. ${ }^{*}\left({ }^{*}\right.$ Money paid to institution.)

\section{References}

1. Kim JM, Romano A, Sanan A, et al. Microsurgical anatomic features and nomenclature of the paraclinoid region. Neurosurgery 2000;46:670-80; discussion $680-72$

2. Raymond J, Guilbert F, Weill A, et al. Long-term angiographic recurrences after selective endovascular treatment of aneurysms with detachable coils. Stroke 2003;34:1398-403

3. Bonita R, Beaglehole R. Recovery of motor function after stroke. Stroke 1988;19:1497-500

4. Rankin J. Cerebral vascular accidents in patients over the age of 60. II. Prognosis. Scott Med J 1957;2:200-15

5. Boet R, Wong GK, Poon WS, et al. Aneurysm recurrence after treatment of paraclinoid/ophthalmic segment aneurysms-a treatment-modality assessment. Acta Neurochir (Wien) 2005;147:611-16; discussion 616

6. Chen L, Kato Y, Karagiozov KL, et al. Usefulness of a simplified management scheme for paraclinoid aneurysms based on a modified classification. Cerebrovasc Dis 2008;26:388-96

7. Fulkerson DH, Horner TG, Payner TD, et al. Endovascular retrograde suction decompression as an adjunct to surgical treatment of ophthalmic aneurysms: analysis of risks and clinical outcomes. Neurosurgery 2009;64:107-11; discussion 111-12

8. Heran NS, Song JK, Kupersmith MJ, et al. Large ophthalmic segment aneurysms with anterior optic pathway compression: assessment of anatomical and visual outcomes after endosaccular coil therapy. J Neurosurg 2007;106: $968-75$

9. Hoh BL, Carter BS, Budzik RF, et al. Results after surgical and endovascular treatment of paraclinoid aneurysms by a combined neurovascular team. $\mathrm{Neu}$ rosurgery 2001;48:78-89; discussion 89-90

10. Hoh BL, Carter BS, Putman CM, et al. Important factors for a combined neurovascular team to consider in selecting a treatment modality for patients with previously clipped residual and recurrent intracranial aneurysms. Neurosurgery 2003;52:732-38; discussion 738-39

11. Iihara K, Murao K, Sakai N, et al. Unruptured paraclinoid aneurysms: a management strategy. J Neurosurg 2003;99:241-47

12. Jin SC, Kwon do H, Ahn JS, et al. Clinical and radiogical outcomes of endovascular detachable coil embolization in paraclinoid aneurysms: a 10-year experience. J Korean Neurosurg Soc 2009;45:5-10

13. Roy D, Raymond J, Bouthillier A, et al. Endovascular treatment of ophthalmic segment aneurysms with Guglielmi detachable coils. AJNR Am J Neuroradiol 1997; 18:1207-15

14. Thornton J, Aletich VA, Debrun GM, et al. Endovascular treatment of paraclinoid aneurysms. Surg Neurol 2000;54:288-99

15. Yadla S, Campbell PG, Grobelny B, et al. Open and endovascular treatment of unruptured carotid-ophthalmic aneurysms: clinical and radiographic outcomes. Neurosurgery 2011;68:1434-43; discussion 1443

16. D'Urso PI, Lanzino G, Cloft HJ, et al. Flow diversion for intracranial aneurysms. A Review. Stroke 2011;42:2363-68 